\title{
RESPUESTA ACOPLADA DE TRASLACIÓN Y TORSIÓN DE ESTRUCTURAS ASIMÉTRICAS INCLUYENDO LA INTERACCIÓN CON EL SUELO
}

\author{
Martha Suarez ${ }^{(1)}$ y Javier Avilés ${ }^{(2)}$
}

\begin{abstract}
RESUMEN
Considerando los efectos de interacción suelo-estructura, se estudia la respuesta acoplada de traslación y torsión de estructuras asimétricas desplantadas sobre un estrato de suelo ante excitación sísmica. El sistema investigado consiste en un oscilador simple torsionalmente acoplado con cimentación enterrada en un estrato blando sobre un semiespacio elástico, sometido a ondas $S H$ con diferentes ángulos de incidencia. Se calculan soluciones numéricas para varias configuraciones del sistema, tomando como movimiento de control el gran temblor de Michoacán de 1985 registrado en tres sitios representativos de las zonas blanda y de transición en el Valle de México. Se evalúan la amplificación dinámica de la excentricidad y la relación entre las fuerzas cortantes acopladas y desacopladas, y se comparan con las actuales recomendaciones reglamentarias para estructuras de edificios (Normas Técnicas Complementarias para Diseño por Sismo del RCDF, Gaceta Oficial Del Distrito Federal, 1995). Se muestra que, al menos para comportamiento lineal de la estructura, las especificaciones de diseño por torsión pueden subestimar la máxima respuesta estructural cuando los efectos de interacción son de excepcional importancia, como sucede en la Ciudad de México.
\end{abstract}

\section{SUMMARY}

By considering the effects of soil-structure interaction, the coupled lateral and torsional response of asymmetric structures placed on a soil layer under seismic excitation is studied. The system investigated consists in a torsionally coupled single oscillator with embedded foundation in a soft stratum over an elastic half-space, subjected to $S H$ waves with different angles of incidence. Both the inertial and kinematic interaction effects are considered. Numerical solutions are calculated for a large number of system configurations, taking as control motion the great 1985 Michoacán earthquake recorded at three sites representative of the transition and soft zones in the Valley of México. The dynamic amplifications of static eccentricity and the base shear ratio between the coupled and uncoupled shears are evaluated and compared with the current code provisions for building structures (Normas Técnicas Complementarias para Diseño por Sismo del RCDF, Gaceta Oficial Del Distrito Federal, 1995). It is shown that, at least for linear behavior of the structure, the design provisions for torsion may underestimate the maximum structural response when the interaction effects are of extreme significance, as happens in Mexico City.

Artículo recibido el 10 de septiembre de 2001 y aprobado para su publicación el 17 de junio de 2002. Se aceptarán comentarios y/o discusiones hasta cinco meses después de su publicación.

(1) Instituto de Ingeniería, UNAM,Ciudad Universitaria, 04510 México, DF, msl@servidor.unam.mx

(2) Instituto Mexicano de Tecnología del Agua, Jiutepec, Morelos, javiles@tajin.imta.mx 


\section{INTRODUCCIÓN}

Algunos artículos en la literatura especializada (Hanh y Liu, 1994; Chandler y Hutchinson, 1987a y b) afirman que diversos reglamentos de construcción del mundo, entre ellos el de la ciudad de México, no consideran adecuadamente las amplificaciones dinámicas que se pueden presentar en estructuras asimétricas cuando son sometidas a excitación sísmica, en especial aquellos edificios con excentricidad estructural pequeña y cuando su periodo de vibración en traslación se acerca al valor de su periodo de vibración en torsión. En las Normas Técnicas Complementarias para Diseño por Sismo del RCDF (Gaceta Oficial Del Distrito Federal, 1995), y en la mayoría de los reglamentos, se especifica que la excentricidad que debe utilizarse en el cálculo de las acciones para el diseño por torsión de los elementos estructurales, debe ser aquélla que considere a las excentricidades natural y accidental, generada la primera por la diferencia en la posición de los centros de masa y rigidez y la segunda por la variación espacial del movimiento del suelo ante el paso de las ondas sísmicas, entre otros factores. La excentricidad de diseño se obtiene mediante la aplicación de fórmulas simples que involucran a la excentricidad estructural y a una dimensión característica de la planta de la estructura, afectadas por coeficientes especificados. Con esto se pretende que su uso en la práctica sea sencillo y que el diseño de las edificaciones se lleve a cabo dentro de niveles de seguridad aceptables.

Entre los estudios llevados a cabo con el propósito de dar fundamento a las especificaciones propuestas en los reglamentos, se encuentran los que únicamente consideran la excentricidad accidental causada principalmente por la rotación de la cimentación ante el paso de las ondas sísmicas (Newmark, 1969; Luco, 1976a y b; Apsel y Luco, 1976; Bielak et al., 1986) y aquellos que tienen en cuenta la excentricidad estructural (Hanh y Liu, 1994; Chopra y Goel, 1991; Chandler y Hutchinson, 1987a y b). Algunos autores han estudiado la respuesta de estructuras considerando su interacción con el suelo (Chandler y Hutchinson, 1987b) pero, generalmente, han omitido la interacción cinemática al considerar sólo la interacción inercial debida a la flexibilidad del suelo, ignorando la diferencia entre el movimiento de campo libre y el movimiento de entrada de la cimentación. Varios autores afirman la necesidad de revisar las expresiones dadas en los reglamentos para obtener las excentricidades de diseño con el propósito de tomar en cuenta, por ejemplo, las demandas de ductilidad del sistema (Bozorgnia y Tso, 1986; Chopra y Goel, 1991; Zhu y Tso, 1992; Tso y Zhu, 1992) y los efectos de interacción en estructuras que tienen periodo fundamental de traslación cercano al valor del periodo fundamental de torsión (Chandler y Hutchinson, 1987a y b). En la mayoría de estos artículos no se consideran los efectos de la profundidad de desplante de la cimentación y no se contemplan estructuras desplantadas sobre suelos estratificados.

El propósito de este trabajo es investigar ambos efectos de la interacción, los cinemáticos e inerciales, en estructuras con movimientos de traslación y torsión acoplados, comparando los resultados con los que se obtienen al aplicar los criterios de diseño propuestos en las normas y señalando los casos que no son cubiertos por éstas. Para ello se utilizan como movimientos de control los acelerogramas registrados en las estaciones $V I V, S C T$ y $C A O$ durante el sismo de Michoacán del 19 de septiembre de 1985. Se consideran trenes de ondas de corte polarizadas horizontalmente que inciden con cierto ángulo con respecto a la vertical. A la estructura se le representa por un oscilador simple con dos grados de libertad, uno de traslación y otro de torsión, 
más tres grados adicionales para la cimentación que surgen al considerar los efectos de la interacción con el suelo. Se analizan estructuras con distintos periodos naturales en traslación y torsión y se calcula su respuesta máxima. Posteriormente se obtienen las excentricidades y cortantes de diseño que son comparadas con las que se estipulan en las normas técnicas. Los cálculos se efectúan en el dominio de la frecuencia, por lo que sólo se contempla el comportamiento lineal de la estructura. Únicamente se considera la incidencia de ondas $S H$ que son las que producen los efectos de torsión cuando inciden con cierto ángulo con respecto a la vertical. Los resultados que se presentan dan una idea de las amplificaciones dinámicas que pueden generarse en edificios, sin embargo, para poder fijar alternativas para modificar las recomendaciones de diseño, se requerirá hacer un estudio más amplio considerando varios registros de temblores y llevando un control estadístico. En este trabajo únicamente se evidencia la necesidad de revisar los criterios reglamentarios.

\section{MODELO}

En este estudio se consideró un modelo formado por un oscilador simple de masa $M$ ubicada a una altura $H_{e}$ y excentricidad $e$, con un grado de libertad en traslación y otro en torsión, como se muestra en la fig. 1. La cimentación es representada por un cilindro de radio $R$, profundidad $D \mathrm{y}$ masa $M_{0}$, que está embebido en un estrato blando de espesor $H_{s}$ y módulo de Poisson $v_{s}=0.45$ apoyado sobre un semiespacio elástico con módulo de Poisson $v_{o}=1 / 3$. La relación de velocidades de ondas de cortante entre el estrato y el semiespacio se tomó como $\beta_{s} / \beta_{o}=0.2$. Al modelo se le sometió a una excitación sísmica dada por ondas de corte con movimiento en la dirección $y$, propagándose en el plano $x-z$ con ángulo $\gamma$ respecto a la vertical, como se aprecia en la fig. 1. Las ecuaciones que definen el movimiento del modelo en el dominio de la frecuencia están dadas por:

$$
\begin{aligned}
& M\left[\ddot{\Delta}_{b}+\ddot{\Delta}_{0}+\ddot{\Delta}_{g}+\left(H_{e}+D\right)\left(\ddot{\varphi}_{0}+\ddot{\varphi}_{g}\right)+e\left(\ddot{\theta}_{b}+\ddot{\theta}_{0}+\ddot{\theta}_{g}\right)\right] \\
& +C_{h h} \dot{\Delta}_{b}+C_{h \theta} e \dot{\theta}_{b}+K_{h h} \Delta_{b}+K_{h \theta} e \theta_{b}=0
\end{aligned}
$$

$+C_{h \theta} e \dot{\Delta}_{b}+C_{\theta \theta} \dot{\theta}_{b}+K_{h \theta} e \Delta_{b}+K_{\theta \theta} \theta_{b}=0$

$$
\begin{aligned}
& M\left[\ddot{\Delta}_{b}+\ddot{\Delta}_{0}+\ddot{\Delta}_{g}+\left(H_{e}+D\right)\left(\ddot{\varphi}_{0}+\ddot{\varphi}_{g}\right)+e\left(\ddot{\theta}_{b}+\ddot{\theta}_{0}+\ddot{\theta}_{g}\right)\right] \\
& +M_{0}\left[\ddot{\Delta}_{0}+\ddot{\Delta}_{g}+E\left(\ddot{\varphi}_{0}+\ddot{\varphi}_{g}\right)+e\left(\ddot{\theta}_{0}+\ddot{\theta}_{g}\right)\right]+F_{x}^{s}=0
\end{aligned}
$$




$$
\begin{aligned}
& M e\left[\ddot{\Delta}_{b}+\ddot{\Delta}_{0}+\ddot{\Delta}_{g}+\left(H_{e}+D\right)\left(\ddot{\varphi}_{0}+\ddot{\varphi}_{g}\right)\right]+J_{\theta}\left(\ddot{\theta}_{b}+\ddot{\theta}_{0}+\ddot{\theta}_{g}\right) \\
& +M_{0} e\left[\ddot{\Delta}_{0}+\ddot{\Delta}_{g}+E\left(\ddot{\varphi}_{0}+\ddot{\varphi}_{g}\right)\right]+J_{\theta}\left(\ddot{\theta}_{0}+\ddot{\theta}_{g}\right)+F_{\theta}^{s}=0 \\
& M\left(H_{e}+D\right)\left[\ddot{\Delta}_{b}+\ddot{\Delta}_{0}+\ddot{\Delta}_{g}+\left(H_{e}+D\right)\left(\ddot{\varphi}_{0}+\ddot{\varphi}_{g}\right)+e\left(\ddot{\theta}_{b}+\ddot{\theta}_{0}+\ddot{\theta}_{g}\right)\right]+J_{\varphi}\left(\ddot{\varphi}_{0}+\ddot{\varphi}_{g}\right) \\
& +M_{0} E\left[\ddot{\Delta}_{0}+\ddot{\Delta}_{g}+E\left(\ddot{\varphi}_{0}+\ddot{\varphi}_{g}\right)+e\left(\ddot{\theta}_{0}+\ddot{\theta}_{g}\right)\right]+J\left(\ddot{\varphi}_{\varphi}+\ddot{\varphi}_{g}\right)+F_{\varphi}^{s}=0
\end{aligned}
$$

donde los subíndices $b, 0$ y $g$ se refieren a la estructura, la cimentación y la excitación efectiva, respectivamente. $\Delta$ у $\varphi$ implican traslaciones a lo largo del eje $y$ y giros alrededor del eje $x$, respectivamente, y $\theta$ indica giros alrededor del eje $z$. Los puntos arriba de estas variables indican derivación con respecto al tiempo. $K$ y $C$ son las rigideces y amortiguamientos estructurales, usando los subíndices $\theta, h$ y $\theta h$ para la torsión, traslación y acoplamiento, respectivamente. $J_{\theta}$ y $J_{\varphi}$ son los momentos de inercia para la torsión y el cabeceo, respectivamente, y $E=D / 2$. Por último, $F_{x}{ }^{s},{F_{\theta}}^{s}$ y $F_{\varphi}{ }^{s}$ están definidas por:

$$
\left[\begin{array}{c}
F_{x}^{s} \\
F_{\varphi}^{s} \\
F_{\theta}^{s}
\end{array}\right]=\left[\begin{array}{ccc}
K_{h}^{s} & K_{m h}^{s} & 0 \\
K_{m h}^{s} & K_{m}^{s} & 0 \\
0 & 0 & K_{+}^{s}
\end{array}\right]\left[\begin{array}{c}
\Delta_{0} \\
\varphi_{0} \\
\theta_{0}
\end{array}\right]
$$

donde $K^{s}$ son las funciones de impedancia del suelo, con subíndices $h, m, t$ y $m h$ que representan traslación, cabeceo, torsión y acoplamiento, respectivamente.

Realizando una normalización conveniente, las ecuaciones de movimiento se pueden rescribir de la siguiente manera:

$$
\begin{aligned}
& \Delta_{b}\left[\frac{\omega_{h}^{2}}{\omega^{2}}+2 i \zeta \frac{\omega_{h}}{\omega}-1\right]-\Delta_{0}-\Delta_{\varphi_{0}}+e_{r} \Delta_{\theta_{b}}\left[\frac{K_{h \theta}}{M \omega^{2}}+\frac{C_{h \theta}}{M \omega} i-1\right]-e_{r} \Delta_{\theta_{0}} \\
& =\Delta_{0 g}\left\{Q_{h}+Q_{\varphi}+e_{r} Q_{\theta}\right\} \\
& e_{r} \Delta_{b}\left[\frac{K_{h \theta}}{M \omega^{2}}+i \frac{C_{h \theta}}{M \omega}-1\right]-e_{r} \Delta_{0}-e_{r} \Delta_{\varphi_{0}}+\Delta_{\theta_{b}} \frac{J_{\theta}}{M R^{2}}\left[\frac{\omega_{\theta}^{2}}{\omega^{2}}+2 i \zeta \frac{\omega_{\theta}}{\omega}-1\right]-\frac{J_{\theta}}{M R^{2}} \Delta_{\theta_{0}} \\
& =\Delta_{0 g}\left\{e_{r} Q_{h}+e_{r} Q_{\varphi}+\frac{J_{\theta}}{M R^{2}} Q_{\theta}\right\}
\end{aligned}
$$




$$
\begin{aligned}
& -\Delta_{b}+\Delta_{0}\left[\frac{K_{h}^{s}}{M \omega^{2}}-\delta_{M}-1\right]+\Delta_{\varphi_{0}}\left[\frac{K_{h m}^{s}}{M R \omega^{2}\left(\delta_{H}+\delta_{D}\right)}-\frac{\delta_{D}}{2\left(\delta_{H}+\delta_{D}\right)} \delta_{M}-1\right]-e_{r} \Delta_{\theta_{b}} \\
& -e_{r} \Delta_{\theta_{0}}\left(\delta_{M}+1\right)=\Delta_{0 g}\left\{Q_{h}\left(\delta_{M}+1\right)+Q_{\varphi}\left[\frac{\delta_{D}}{2\left(\delta_{H}+\delta_{D}\right)} \delta_{M}+1\right]+e_{r} Q_{\theta}\left(\delta_{M}+1\right)\right\} \\
& -e_{r} \Delta_{b}-e_{r} \Delta_{0}\left(\delta_{M}+1\right)-e_{r} \Delta_{\varphi_{0}}\left[\frac{\delta_{D}}{2\left(\delta_{H}+\delta_{D}\right)} \delta_{M}+1\right]-\frac{J_{\theta}}{M R^{2}} \Delta_{\theta_{b}}+\Delta_{\theta_{0}}\left[\frac{K_{t}^{s}}{M R^{2} \omega^{2}}\right. \\
& \left.-\left(\delta_{M}+1\right) \frac{J_{\theta}}{M R^{2}}\right]=\Delta_{0 g}\left\{e_{r} Q_{h}\left(\delta_{M}+1\right)+e_{r} Q_{\varphi}\left[\frac{\delta_{D}}{2\left(\delta_{H}+\delta_{D}\right)} \delta_{M}+1\right]+\frac{J_{\theta}}{M R^{2}} Q_{\theta}\left(\delta_{M}+1\right)\right\} \\
& -\Delta_{b}+\Delta_{0}\left[\frac{K_{h m}^{s}}{M R^{2} \omega^{2}\left(\delta_{H}+\delta_{D}\right)}-\frac{\delta_{D}}{2\left(\delta_{H}+\delta_{D}\right)} \delta_{M}-1\right]+\Delta_{\varphi_{0}}\left[\frac{K_{m}^{s}}{M R^{2} \omega^{2}\left(\delta_{H}+\delta_{D}\right)^{2}}\right. \\
& \left.-\frac{J_{\varphi}}{M R^{2}} \frac{1}{\left(\delta_{H}+\delta_{D}\right)^{2}}\left(\delta_{M}+1\right)-\frac{\delta_{D}^{2}}{4\left(\delta_{H}+\delta_{D}\right)^{2}} \delta_{M}-1\right]-e_{r} \Delta_{\theta_{b}} \\
& -e_{r} \Delta_{\theta_{0}}\left[\frac{\delta_{D}}{2\left(\delta_{H}+\delta_{D}\right)} \delta_{M}+1\right]=\Delta_{0 g}\left\{Q_{h}\left[\frac{\delta_{D}}{2\left(\delta_{H}+\delta_{D}\right)} \delta_{M}+1\right]\right. \\
& \left.+Q_{\varphi}\left[\frac{J_{\varphi}}{M R^{2}\left(\delta_{H}+\delta_{D}\right)^{2}}\left(\delta_{M}+1\right)+\frac{\delta_{D}^{2}}{4\left(\delta_{H}+\delta_{D}\right)^{2}} \delta_{M}+1\right]+e_{r} Q_{\theta}\left[\frac{\ddot{a}_{D}}{2\left(\delta_{H}+\delta_{D}\right)} \delta_{M}+1\right]\right\}
\end{aligned}
$$

donde $\omega_{h}=\left(K_{h} / M\right)^{1 / 2}, \omega_{\theta}=\left(K_{\theta} / J_{\theta}\right)^{1 / 2}, \omega=2 \pi f(f=$ frecuencia $), \delta_{M}=M_{0} / M, \delta_{D}=D / R, \delta_{H}=H_{e} / R \quad \mathrm{y}$ $e_{r}=e / R$. $Q_{h}=\Delta_{g} / \Delta_{0 g}, Q_{\varphi}=\left(H_{e}+D\right) \varphi_{g} / \Delta_{0 g}$ y $Q_{\theta}=R \theta_{g} / \Delta_{0 g}$ son las relaciones de los movimientos de entrada de la cimentación con respecto a la amplitud $\Delta_{0 g}$ del movimiento horizontal de campo libre y $\zeta=0.05$ es el amortiguamiento de la estructura.

\section{RIGIDECES DINÁMICAS Y EXCITACIONES EFECTIVAS}

En el modelo utilizado para este estudio se consideran diferentes profundidades de desplante $D$ de la cimentación y espesores $H_{s}$ del estrato. Para obtener la respuesta a la traslación y torsión de la estructura, se requiere conocer previamente las rigideces dinámicas del suelo y los movimientos de entrada de la cimentación, ambos dependientes de la frecuencia de excitación, para los diferentes valores de $D / R$ y $H_{s} / R$ seleccionados. Las primeras representan a los resortes y amortiguadores del suelo, mientras que los segundos se refieren a los movimientos de campo libre modificados por la presencia de la cimentación que, al ser rígida, actúa como un elemento promediador de éstos. Las funciones de impedancia o rigideces dinámicas se calcularon usando 


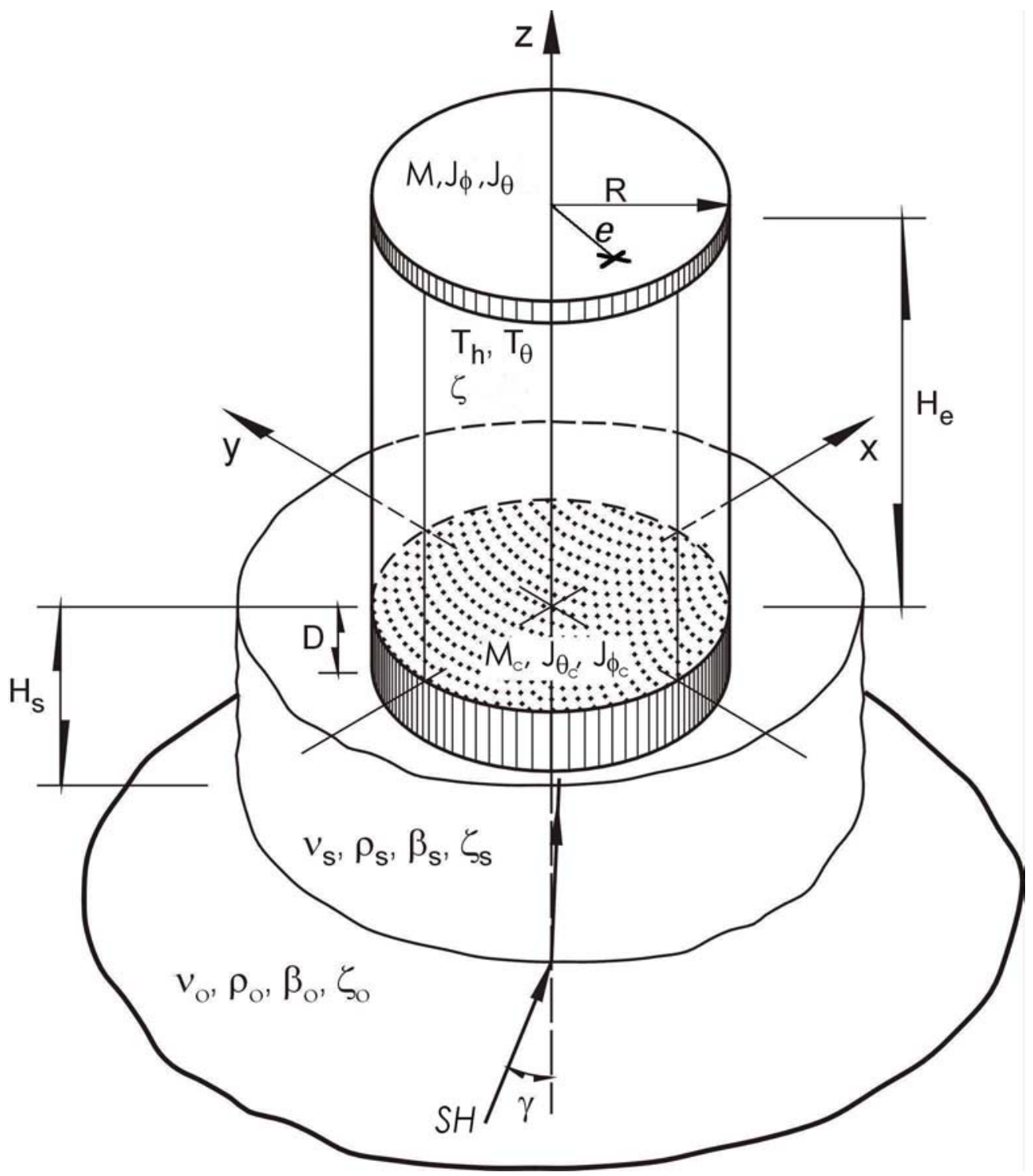

Figura 1. Características del modelo del sistema suelo-estructura utilizado en el estudio.

un método de elementos finitos basado en la técnica del estrato delgado (Tassoulas y Kausel, 1983). Este método sólo admite una formación estratigráfica horizontal apoyada sobre base rígida. Para evitar el rebote de las ondas debido a la presencia de esta base y simular el efecto de su radiación al infinito, se consideraron dos estratos. El real de espesor $H_{s}$ que aloja a la cimentación y uno ficticio con el doble de espesor para asegurar que las ondas reflejadas por su base sean de amplitud despreciable, actuando de esta manera como si fuera un semiespacio. Las excitaciones efectivas se determinaron utilizando una técnica propuesta por Iguchi (1982), la cual requiere de las funciones de impedancia y de los movimientos y tracciones de campo libre para su aplicación. Estos últimos se obtienen empleando métodos bien conocidos en la teoría de propagación de ondas (Achenbach, 1976). 


\begin{tabular}{|c|c|c|c|c|c|c|}
\hline \multicolumn{7}{|c|}{$\begin{array}{l}\text { Tabla 1. Rigideces estáticas para cimentaciones superficiales }(D / R=0) \text { y enterradas } \\
(D / R=1) \text { en un estrato de suelo con profundidad variable }\left(H_{s} / R=2,3 \text { y } 5\right) \text {. }\end{array}$} \\
\hline & \multicolumn{3}{|c|}{$D / R=0$} & \multicolumn{3}{|c|}{$D / R=1$} \\
\hline $\boldsymbol{H}_{s} / \boldsymbol{R}$ & $K_{h}^{0} / G R$ & $K_{m}{ }^{0} / G R^{3}$ & $K_{t}^{0} / G R^{3}$ & $K_{h}^{0} / G R$ & $K_{m}{ }^{0} / G R^{3}$ & $K_{t}^{0} / G R^{3}$ \\
\hline 2 & 5.5063 & 4.5393 & 5.8697 & 14.2950 & 17.8420 & 21.1594 \\
\hline 3 & 5.2618 & 4.5222 & 6.0672 & 11.8310 & 15.3730 & 20.5169 \\
\hline 5 & 5.2078 & 4.7850 & 6.5710 & 10.5360 & 14.7140 & 20.7462 \\
\hline
\end{tabular}

En las figs. 2 y 3 se grafican los coeficientes de rigidez $k^{s}$ y amortiguamiento $c^{s}$ de las funciones de impedancia expresadas como $K^{s}=K^{o}\left(k^{s}+i \eta c^{s}\right)\left(1+i 2 \zeta_{s}\right)$ para los modos de traslación, cabeceo y torsión de la cimentación, siendo $\zeta_{s}=0.05$ el amortiguamiento del estrato; los valores de las rigideces estáticas $K^{o}$ se indican en la tabla 1. Debido a que el espesor del estrato influye en la rigidez total del sistema, el valor de la rigidez estática también se ve afectado. A diferencia de lo que ocurre para estratos sobre base rígida en donde es posible definir una función lineal que tome en cuenta el espesor y las propiedades mecánicas del suelo para calcular de manera aproximada la rigidez estática (Kausel y Roësset, 1975), para estratos sobre un semiespacio no parece existir una correlación lineal. Los resultados de la fig. 2 corresponden a cimentaciones superficiales $(D / R=0)$, mientras que los de la fig. 3 a cimentaciones enterradas $(D / R=1)$, para diferentes valores del espesor del estrato, a saber: $H_{s} / R=2,3$ y 5 . Estos resultados muestran variaciones con respecto a la frecuencia adimensional $\eta=\omega R / 2 \pi \beta_{s}$ que se deben principalmente a que la frecuencia natural del estrato varía en proporción inversa a su espesor. Esto se ve reflejado en la posición de los valles que se presentan en las frecuencias resonantes del estrato como se aprecia en las gráficas de las figs. 2 y 3, y en la ausencia de amortiguamiento por radiación para frecuencias por debajo de la frecuencia fundamental del estrato. Este efecto es más evidente para el modo de cabeceo que para el de traslación horizontal y el de torsión, pero tiende a ser pequeño para cimientos enterrados, como se aprecia en los resultados aquí presentados (figs. 2 y 3). Para frecuencias menores que la primera frecuencia resonante del estrato, los valores del amortiguamiento son muy bajos puesto que reflejan la pérdida de energía sólo por amortiguamiento histerético. Destacan también las reducciones de los coeficiente de rigidez con la frecuencia de excitación debido a la influencia que tiene ésta en la inercia del suelo, así como las oscilaciones de los coeficientes de rigidez y amortiguamiento debido a las frecuencias naturales del suelo que son más pronunciadas para estratos de espesores pequeños y prácticamente no se presentan para el cabeceo. Si el amortiguamiento del suelo fuera nulo, los valles de estas gráficas se prolongarían hasta tomar el valor nulo.

En la fig. 4 se exhiben las excitaciones efectivas para traslación, cabeceo y torsión debidas al arribo de ondas de cortante con distintos ángulos de incidencia. Para cimentaciones superficiales, se aprecia que sólo en el caso de incidencia vertical puede considerarse que no existe interacción cinemática, esto es, la excitación efectiva de la cimentación es igual al movimiento de campo libre. Para los demás casos, asumir esto conduciría a resultados erróneos. Como los resultados están normalizados con respecto a las amplitudes del movimiento de campo 
libre, los efectos del espesor del estrato se cancelan, de suerte que los valores aquí proporcionados son aplicables a estratos de cualquier tamaño.

\section{AMPLIFICACIONES DINÁMICAS MÁXIMAS}

Para conocer los efectos en la respuesta a la torsión cuando se considera la interacción cinemática, se analizaron y compararon las respuestas de sistemas con profundidad de desplante nula para diferentes ángulos de incidencia. Posteriormente se compararon los sistemas con $D / R=0$ e incidencia vertical de ondas sísmicas con aquellos desplantados a $D / R=1 \mathrm{y}$, finalmente, los que están enterrados en el estrato para distintas características del modelo. Para ello se asumió una relación de densidades entre la estructura y el suelo de $\delta_{\rho}=0.15 ; \zeta_{s}=\zeta_{0}=0.05, \delta_{M}=0.25, v_{s}=0.45$, $v_{o}=0.33 \mathrm{y}$, como movimientos de excitación, los registros del sismo de Michoacán en las estaciones $C A O, S C T$ y $V I V$, localizadas las dos primeras en la zona del lago y la última en la zona de transición de la Ciudad de México. Las amplificaciones de los acelerogramas empleados se muestran en la fig. 5. En los cálculos se consideraron los periodos dominantes del terreno en estos sitios, esto es: $T_{s}=3.5,2$ y $0.6 \mathrm{~s}$, respectivamente.

Se analizaron varios osciladores con periodo natural a la traslación de $T_{h}=0.5,1,2$ y $3 s$, para un amplio rango de valores de la relación $\lambda=T_{h} / T_{\theta}$ entre el periodo de traslación con respecto al de torsión. Se calculó su respuesta máxima a la traslación y a la torsión, $\Delta_{b}{ }^{\max } \mathrm{y} \Delta_{\theta}^{\max }$, obteniendo la razón de las fuerzas cortantes y la amplificación dinámica de la excentricidad de diseño de la siguiente manera:

$$
\frac{V_{h}}{V_{h}^{\text {des }}}=\frac{\Delta_{b}^{\max }}{S}
$$

$e_{r}^{d i s}=\frac{K_{\theta}}{K_{h}} \frac{\Delta_{\theta}}{S} \frac{1}{R^{2}}=\left(\frac{\lambda^{2}}{2} \frac{\Delta_{\theta}}{S}\right)$

donde $V_{h}=K_{h} \Delta_{b}{ }^{\max }$ es el cortante de diseño del sistema en estudio y $V_{h}=K_{h} S$, siendo $S$ el espectro de desplazamientos para el oscilador con respuestas a la torsión y traslación desacopladas $\left(e_{r}=0\right)$; $e_{r}{ }^{d i s}$ es la excentricidad de diseño normalizada con respecto al radio. El momento de torsión para el que se necesitan diseñar los elementos estructurales, está dado por

$F_{\theta}^{t}=F_{\theta}^{e}+F_{\theta}^{a}$

donde $F_{\theta}^{e}$ y $F_{\theta}^{a}$ son los momentos torsionantes natural y accidental, respectivamente. Según las Normas Técnicas Complementarias para Diseño por Sismo del RCDF, $F_{\theta}^{e}=\left(1.5 e_{r}\right)\left(R V_{h}^{\text {des }}\right) \mathrm{y}$ $F_{\theta}^{e}=B\left(0.1 V_{h}^{\text {des }}\right)$, donde $B$ es la dimensión de la planta del cimiento en la dirección de la excentricidad; en el caso del modelo en estudio $B=2 R$. Con base en esto, se puede afirmar que si no hubiera excentricidad estructural, el cortante desacoplado debería ser igual al de diseño, y la 

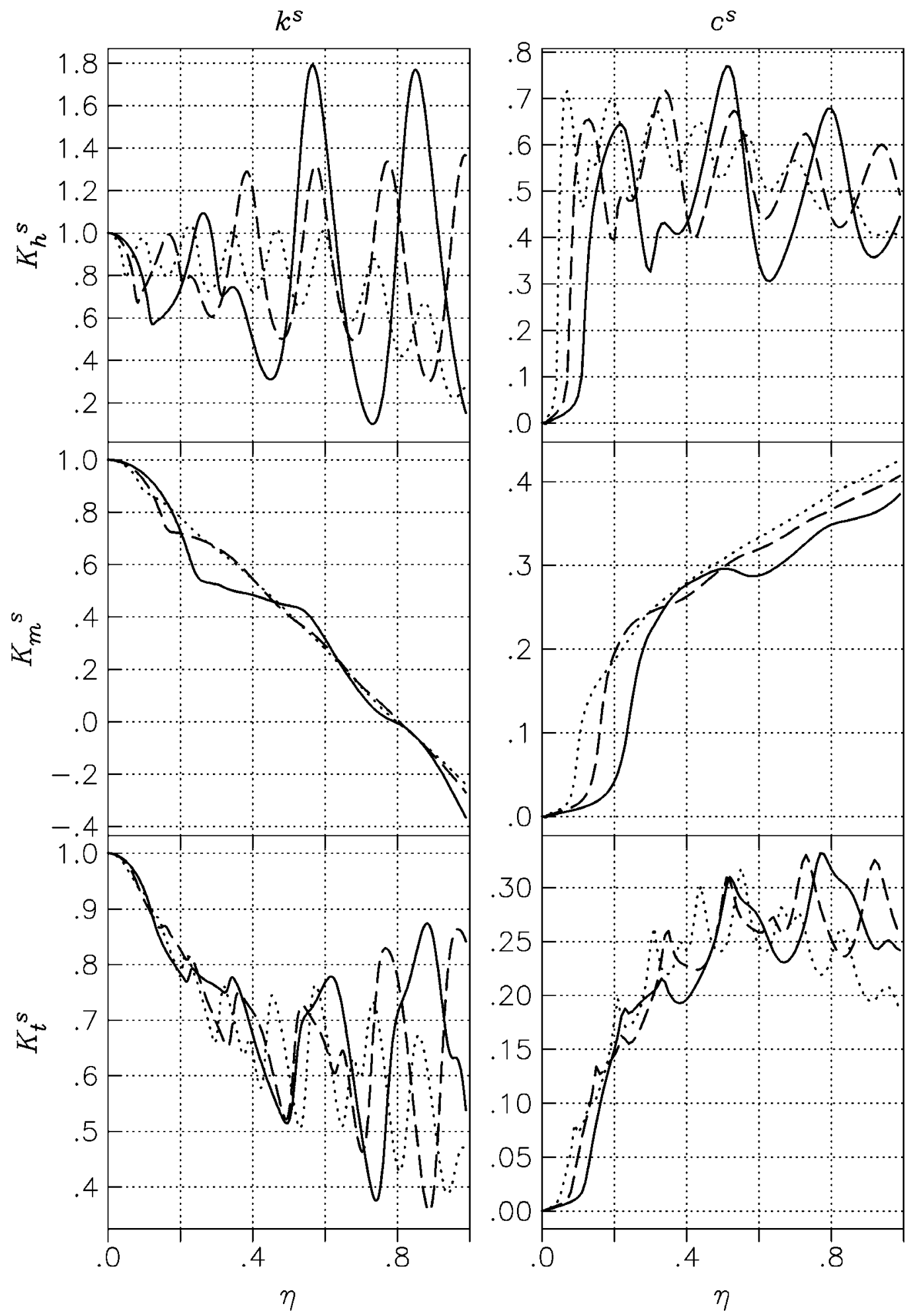

Figura 2. Funciones de impedancia para una cimentación con $D / R=0$ en un estrato con $H_{s} / R=2$ (línea continua), $H_{s} / R=3$ (línea a trazos) y $H_{s} / R=5$ (línea punteada). 

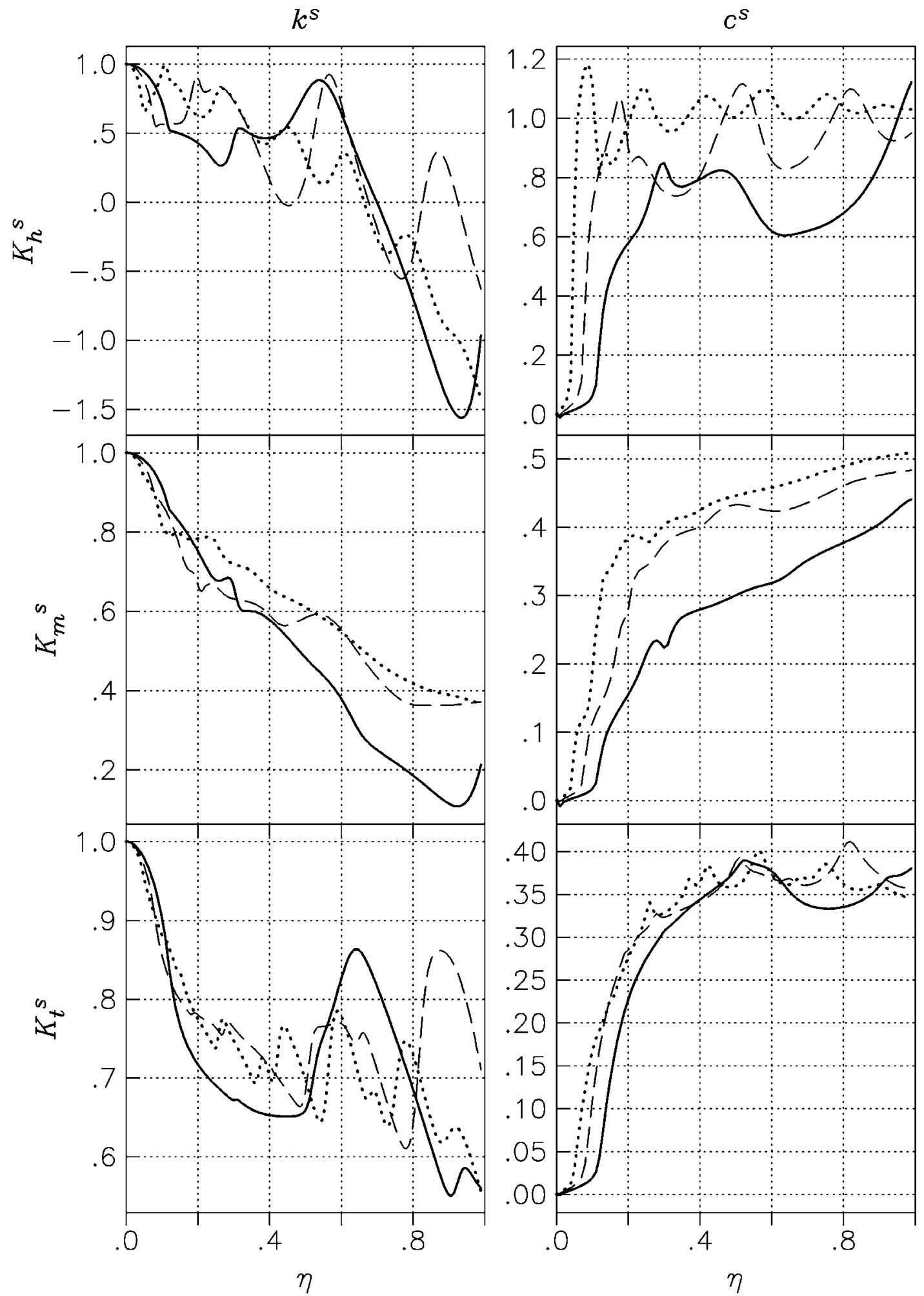

Figura 3. Funciones de impedancia para una cimentación con $D / R=1$ en un estrato con $H_{S} / R=2$ (línea continua), $H_{s} / R=3$ (línea a trazos) y $H_{s} / R=5$ (línea punteada). 

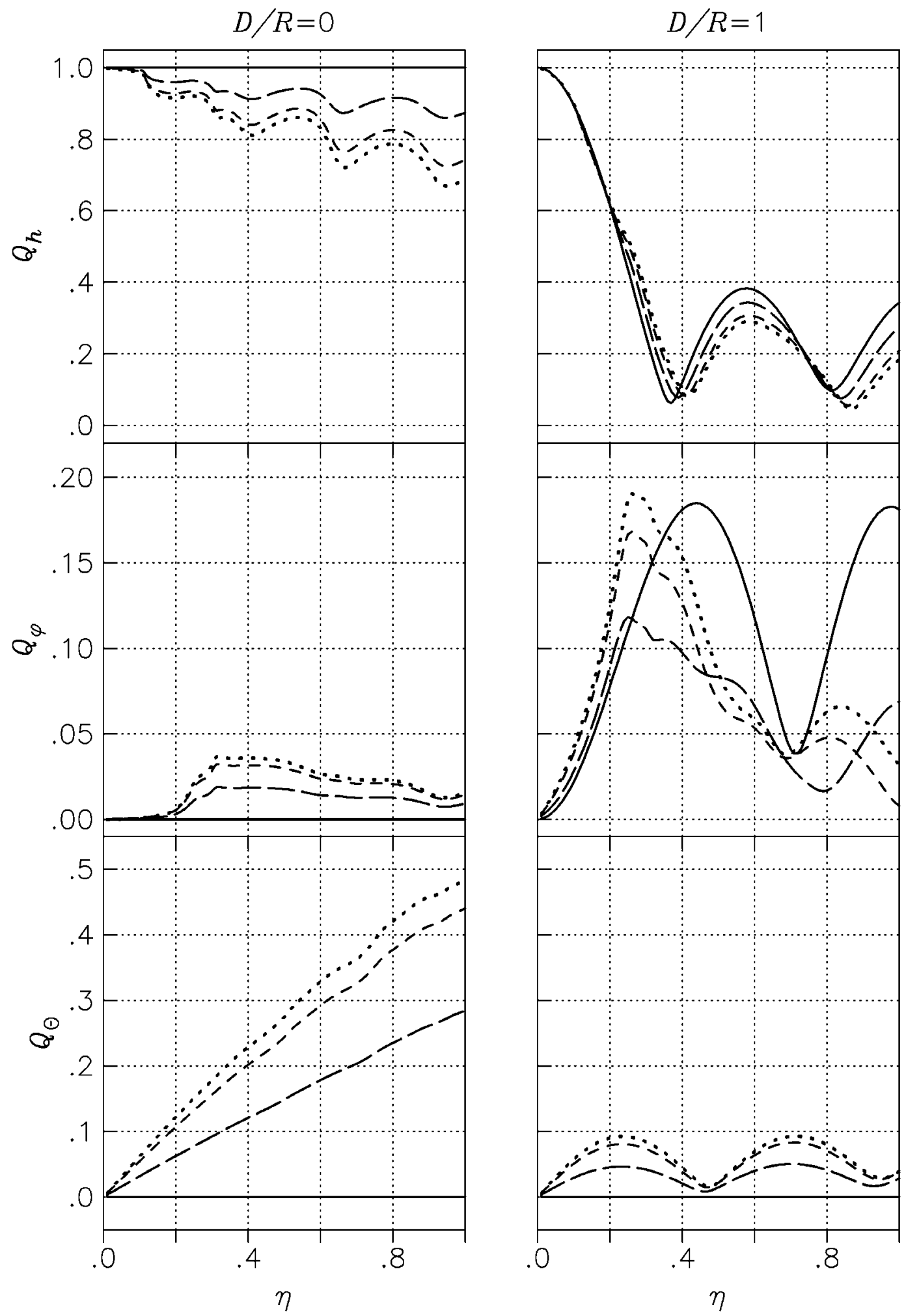

Figura 4. Movimientos de entrada considerando ángulo de incidencia $\gamma=0^{\circ}$ (línea continua), $\gamma=30^{\circ}$ (trazos largos), $\gamma=60^{\circ}$ (trazos pequeños) y $\gamma=90^{\circ}$ (línea punteada). 

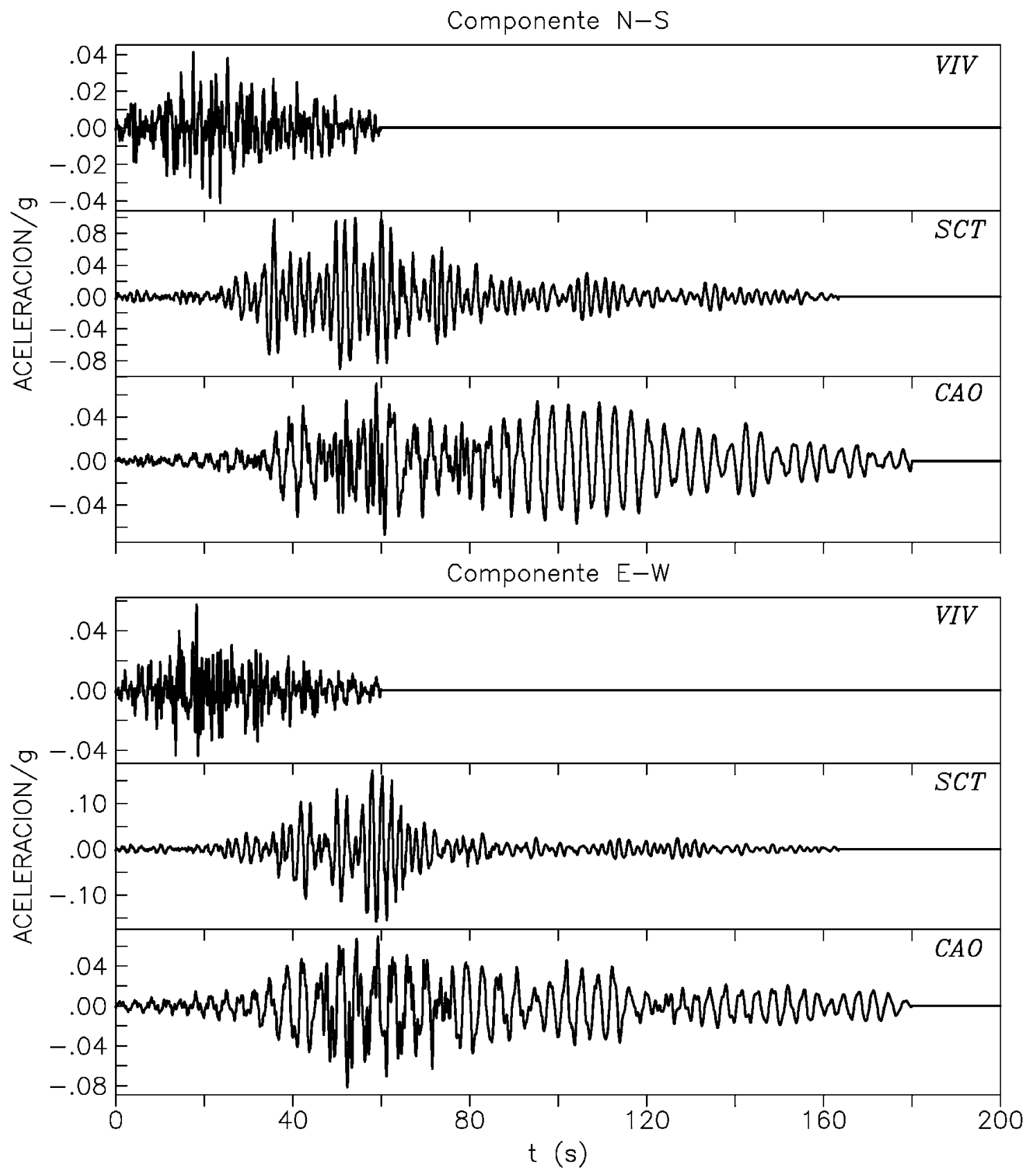

Figura 5. Acelerogramas registrados en las estaciones VIV, SCT y CAO para el sismo de Michoacán del 19 de septiembre de 1985.

torsión se generaría al actuar éste en una posición diferente de la del centro de masa que, según el reglamento, se ubica a $0.1 B(0.2 R)$. En una estructura asimétrica, si la torsión accidental tomara el valor nulo, la torsión estructural sería la generada por el cortante acoplado multiplicado por la excentricidad natural o, como lo sugieren las normas, sería igual al cortante desacoplado $V_{h}^{d e s}$ multiplicado por el $150 \%$ del valor de la excentricidad estática. Debido a que para la obtención de los momentos torsionantes con las fórmulas empíricas propuestas en el RCDF se requieren el 
cortante basal desacoplado y la excentricidad natural, una manera de apreciar las diferencias que existen en relación con aquellos calculados directamente resolviendo sistemas de ecuaciones, es comparando los cocientes entre la excentricidad de diseño y la natural, y entre los cortantes basales acoplado y desacoplado. Algunos autores (Rosenblueth y Elorduy, 1969; Chandler y Hutchinson, 1987a y b) presentan gráficas donde muestran la amplificación dinámica de la excentricidad y la relación de cortantes basales acoplado entre desacoplado en función del parámetro $\lambda$. En la fig. 6 se reproducen las gráficas de Rosenblueth y Elorduy (1969) para la condición de base rígida. En ellas se observa que aunque para la mayoría de los valores de $\lambda$ el cortante acoplado es prácticamente igual al desacoplado y la amplificación dinámica de la excentricidad tiende a 1.15 cuando $\lambda$ crece, la excentricidad dinámica puede llegar a ser mayor que 2.5 veces la estructural y el cortante acoplado casi un $30 \%$ menor que el desacoplado para $\lambda \approx 1$. Suponiendo que las torsiones accidentales fueran nulas, los efectos más desfavorables originados cuando los periodos naturales de traslación y torsión son iguales cumplen aproximadamente con la recomendación reglamentaria, esto es:

$$
F_{\theta}^{t}=1.5 e_{r} V_{h}^{d e s}=e_{r}^{d i s} V_{h}
$$

de donde

$$
1.5=\frac{e_{r}^{d i s}}{e_{r}} \frac{V_{h}}{V_{h}^{\text {des }}}
$$

Para el caso más desfavorable de la fig. 6 , cuando $\lambda \approx 1$, el producto de las amplificaciones dinámicas de la excentricidad con el cociente de los cortantes da un valor superior a 1.5 (aproximadamente 2) que puede quedar cubierto al tomar en cuenta las torsiones accidentales. Sin embargo, hasta donde los autores de este texto tienen conocimiento, no se han analizado los criterios de diseño cuando se considera la interacción cinemática.

En las figs. 7 y siguientes, para que los valores del cortante y de la excentricidad dinámica calculados de manera rigurosa coincidan con lo que se propone en normas, la tendencia del valor para las relaciones de $V_{h} / V_{h}^{d e s}$ y $e_{r}{ }^{d i s} / e_{r}$ deben ser de la unidad en el primer caso y no mayores de 1.5 en el segundo, para cualquier valor de la relación de periodos de traslación al de torsión, $\lambda$, siempre y cuando la torsión accidental sea nula. Muchos de los sistemas estructurales analizados en este trabajo están dentro del comportamiento que se predice al aplicar las normas; sin embargo, existen algunos casos que quedan desprotegidos. En la fig. 7 se presentan los resultados obtenidos para osciladores con periodo a la traslación $T_{h}=1 \mathrm{~s}$, esbeltez $H_{e} / R=1$ y excentricidad $e_{r} / R=0.10$, desplantados sobre la superficie de un estrato de espesor $H_{S} / D=2$ ante excitaciones sísmicas con ángulos de incidencia $\gamma=0^{\circ}, 30^{\circ}, 60^{\circ}$ y $90^{\circ}$. Se observa que el pico donde se presenta la máxima amplificación dinámica de la excentricidad varía dependiendo de las características del sitio y de las del oscilador y en algunos casos se presentan dos picos ( $V I V$ y $C A O)$. El pico no siempre se localiza cuando el valor de $\lambda$ es cercano a la unidad, sino que por la flexibilidad del suelo llega a aparecer incluso para valores tan pequeños. 

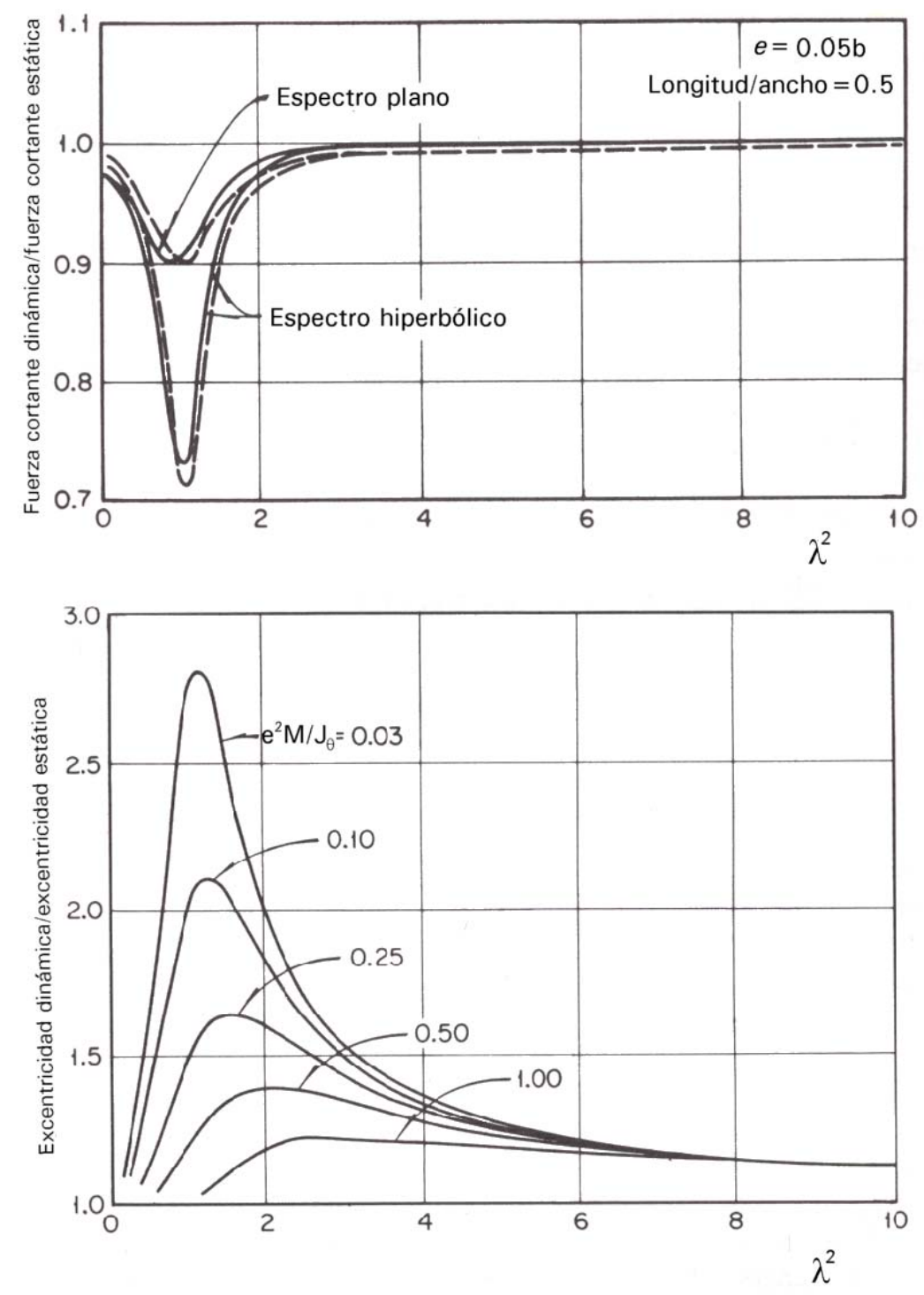

Figura 6. Relación entre fuerzas cortantes dinámicas y estáticas, y factor de amplificación para la excentricidad (modificada a partir de Newmark y Rosenblueth, 1971) .

como $\lambda=0.3(C A O)$ y con amplificaciones de la excentricidad superiores a $4(S C T)$. Pese a que el cortante acoplado tiende a valores menores que el del desacoplado para los valores de $\lambda$ donde se presentan valores altos de la amplificación dinámica de la excentricidad, lo que implica que la torsión natural $\left(F_{\theta}^{e}\right)$ está en cierta medida compensada, para el caso de estructuras desplantadas sobre suelo blando $(C A O)$, esta compensación parece insuficiente debido al alto valor de la amplificación de la excentricidad y lo cercano del cociente entre cortantes a la unidad, incluso para algunas estructuras con $\lambda=0.5$ sometidas al acelerograma registrado en $S C T$, el cociente del cortante acoplado sobre el desacoplado da por resultado la unidad. También se observa que la influencia del ángulo de incidencia prácticamente es nula debido al fuerte contraste de impedancias entre el semiespacio y el estrato, que tiende a refractar las ondas a un ángulo cercano 
a la posición vertical. Se nota que, grosso modo, para valores $\lambda \geq 1.2$ los criterios utilizados en el reglamento son adecuados e incluso conservadores. Sin embargo, para valores $\lambda<1.2$ esto no sucede ni siquiera para el caso de suelos poco flexibles (VIV). Por otro lado, la aseveración de $V_{=} V_{h}^{d e s}$ parece ser adecuada en estos casos.

Los resultados para osciladores con $H_{e} / R=3, e_{r} / R=0.10$ y $D / R=1$, desplantados en estratos con diferentes espesores $\left(H_{S} / D=2,3\right.$ y 5$)$ se muestran en la fig. 8. Aquí se observa que el valor de $\lambda$ donde se presentan los picos de la amplificación de la excentricidad de diseño depende básicamente de la rigidez de le estructura y del espesor del estrato que también afecta al valor de la amplitud. Su influencia se aprecia mejor para estructuras cuyos periodos naturales de vibrar no son muy pequeños o muy grandes $\left(T_{h} \neq 0.5\right.$ y $\left.3 \mathrm{~s}\right)$. La afirmación de otros autores (Chandler y Hutchinson, 1987a y b; Bielak et al., 1986) en el sentido de que las mayores amplificaciones dinámicas ocurrían cuando el valor del periodo desacoplado a la traslación era muy parecido al de torsión de la estructura, es válida si el análisis se lleva a cabo en estructuras desplantadas sobre un semiespacio, para estructuras sobre medios estratificados esto puede no ocurrir.

La influencia de la profundidad de desplante se aprecia en la fig. 9. Los cálculos se efectuaron para estructuras con $H_{e} / R=3, T_{h}=2 s$ y $e_{r} / R=0.10$, empotradas en estratos de espesor $H_{s} / R=2$ y 5 , con profundidad de desplante $D / R=0$ y 1 . De los resultados obtenidos se desprende que la influencia de la profundidad de desplante es poco significativa en la respuesta. Las diferencias, en cuanto a lo que se propone en las normas, se presentan para la amplificación de la excentricidad y el cociente de los cortantes, llegando a tener valores de más del $300 \%$ en el primer caso $(C A O)$ y difiriendo en más de un $25 \%$ para el segundo $(V I V)$.

La influencia del periodo de la estructura a la traslación se muestra en la fig. 10. Aquí se grafican los resultados para osciladores con $H_{e} / R=1, e_{r} / R=0.10$ y $D / R=0$ y con $T_{h}=0.5,1,2$ y $3 s$, desplantados en estratos de espesores $H_{S} / R=2,3$ y 5 y sometidos a la aceleración del registro de $S C T$. Los valores de $\lambda$ donde se presentan los picos de las amplificaciones dinámicas varían dependiendo del periodo natural de traslación de la estructura, correspondiendo los valores más pequeños a las estructuras más rígidas. El número de picos que se presenten en las gráficas dependerá del tipo de suelo en donde esté cimentada la estructura como se aprecia en la fig. 11, en donde se analizan osciladores con las mismas características descritas arriba, pero sometidos a la aceleración del registro de $C A O$.

En la fig. 12 se graficaron los resultados para diferentes valores de esbeltez de la estructura $\left(H_{e} / R=1\right.$ y 3$)$ y de su rigidez $\left(T_{h}=1\right.$ y $\left.2 s\right)$, considerando $H_{s} / R=3, D / R=1$ y $e_{r} / R=0.10$. Es evidente con estos resultados que uno de los parámetros que más afecta la respuesta de la estructura es su periodo natural de vibrar, la esbeltez afecta en la amplitud de los picos de la amplificación dinámica de la excentricidad y en la posición del segundo pico. Al igual que en los casos descritos arriba, existe un rango de valores de $\lambda$ que dependen básicamente de las características del sistema para los cuales los lineamientos especificados en las normas no se cumplen. Además, en la figura se observa que el cortante acoplado puede llegar a ser superior al desacoplado hasta en un $20 \%$ para el mismo valor de $\lambda$ para el que las amplificaciones dinámicas de la excentricidad son altas, lo que deriva en subestimar los valores de la torsión para la que se deben diseñar esas estructuras. 

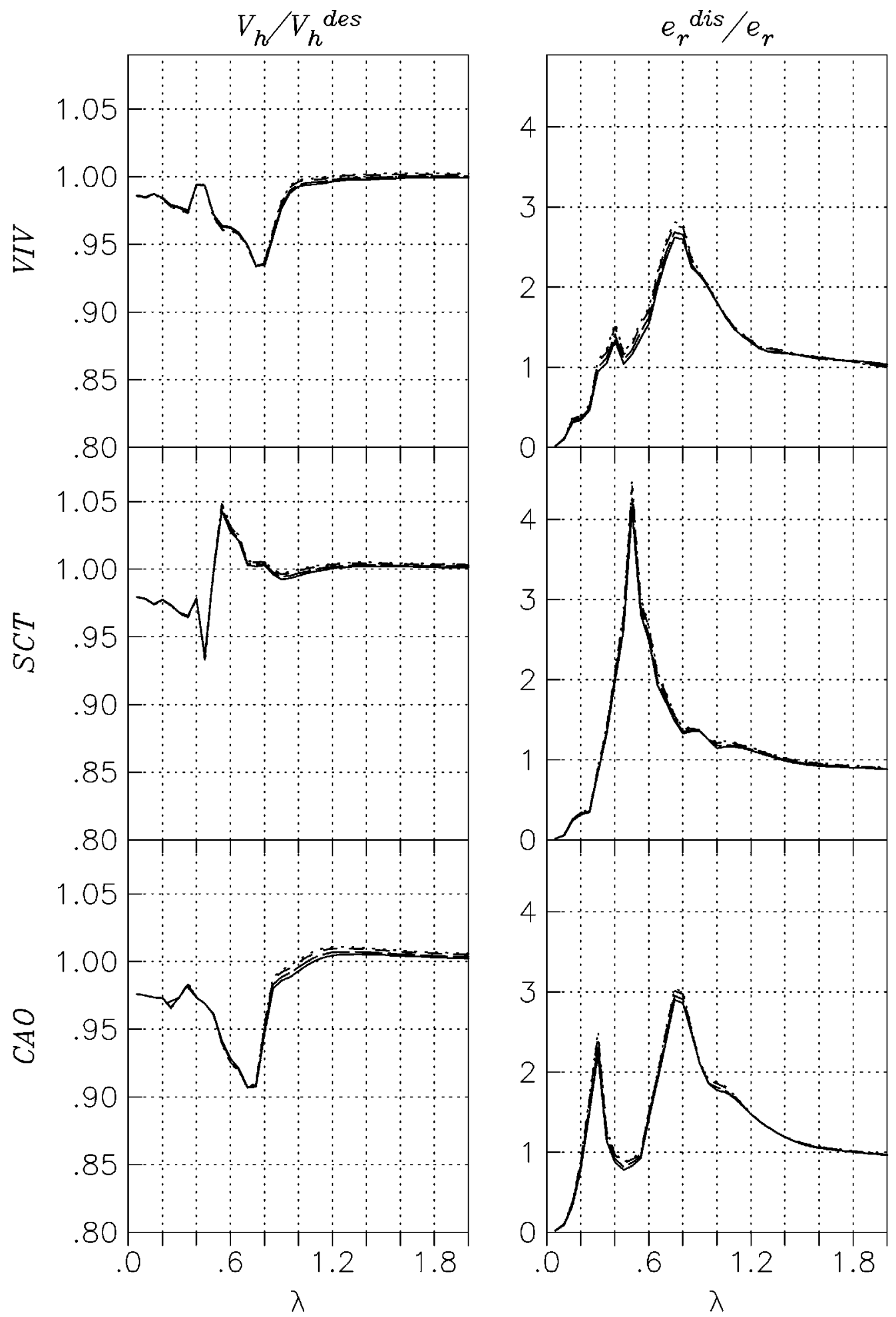

Figura 7. Resultados para osciladores sometidos a la incidencia de ondas $S H$ con ángulo $\gamma=0^{\circ}$ (línea continua), $\gamma=30^{\circ}$ (trazos largos), $\gamma=60^{\circ}$ (trazos pequeños) y $\gamma=90^{\circ}$ (línea punteada). 

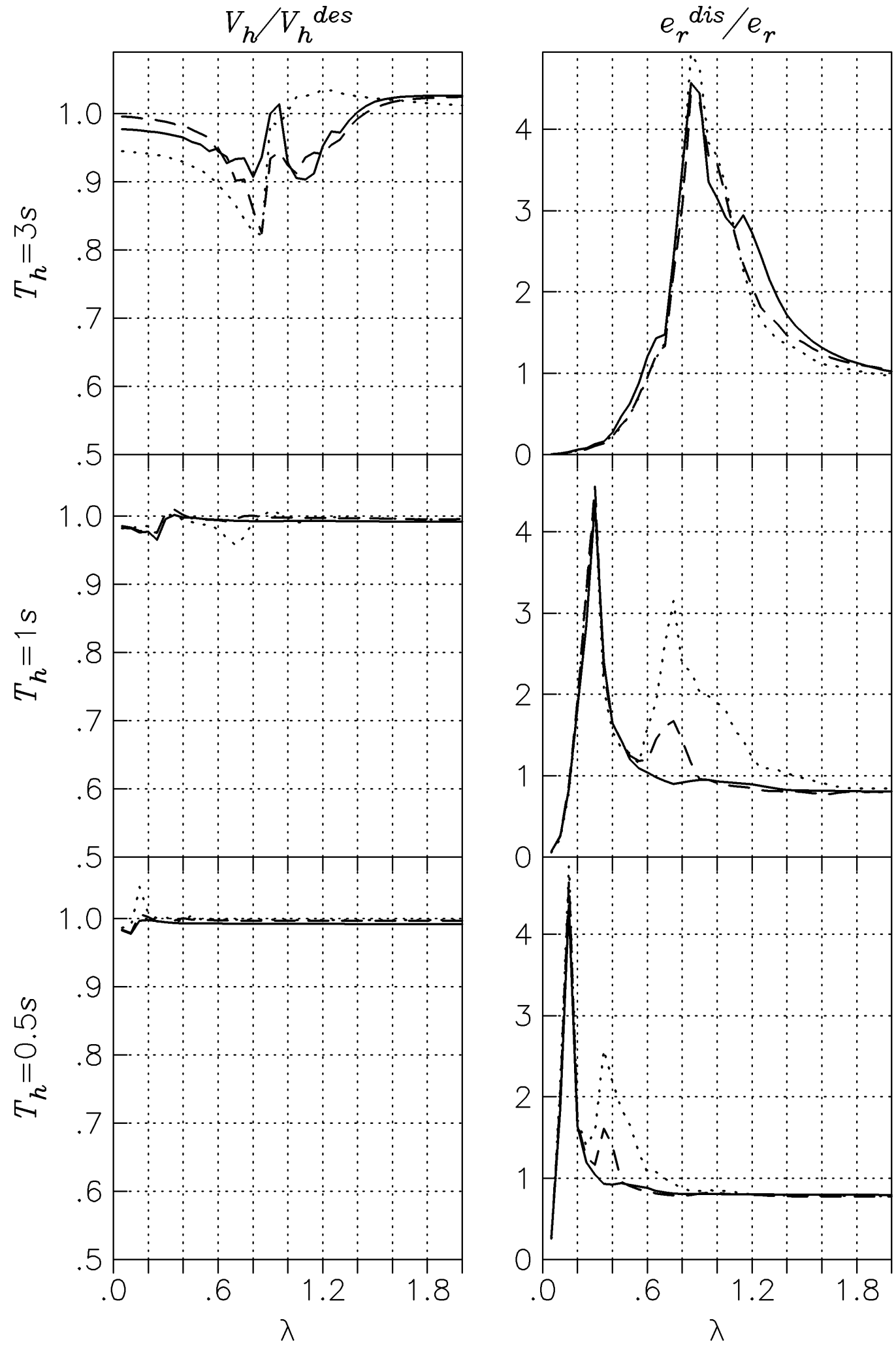

Figura 8. Resultados para osciladores desplantados en un estrato de espesor $H_{s} / R=2$ (línea continua), $H_{S} / R=3$ (trazos largos) y $H_{s} / R=5$ (línea punteada) usando el registro de $C A O$. 

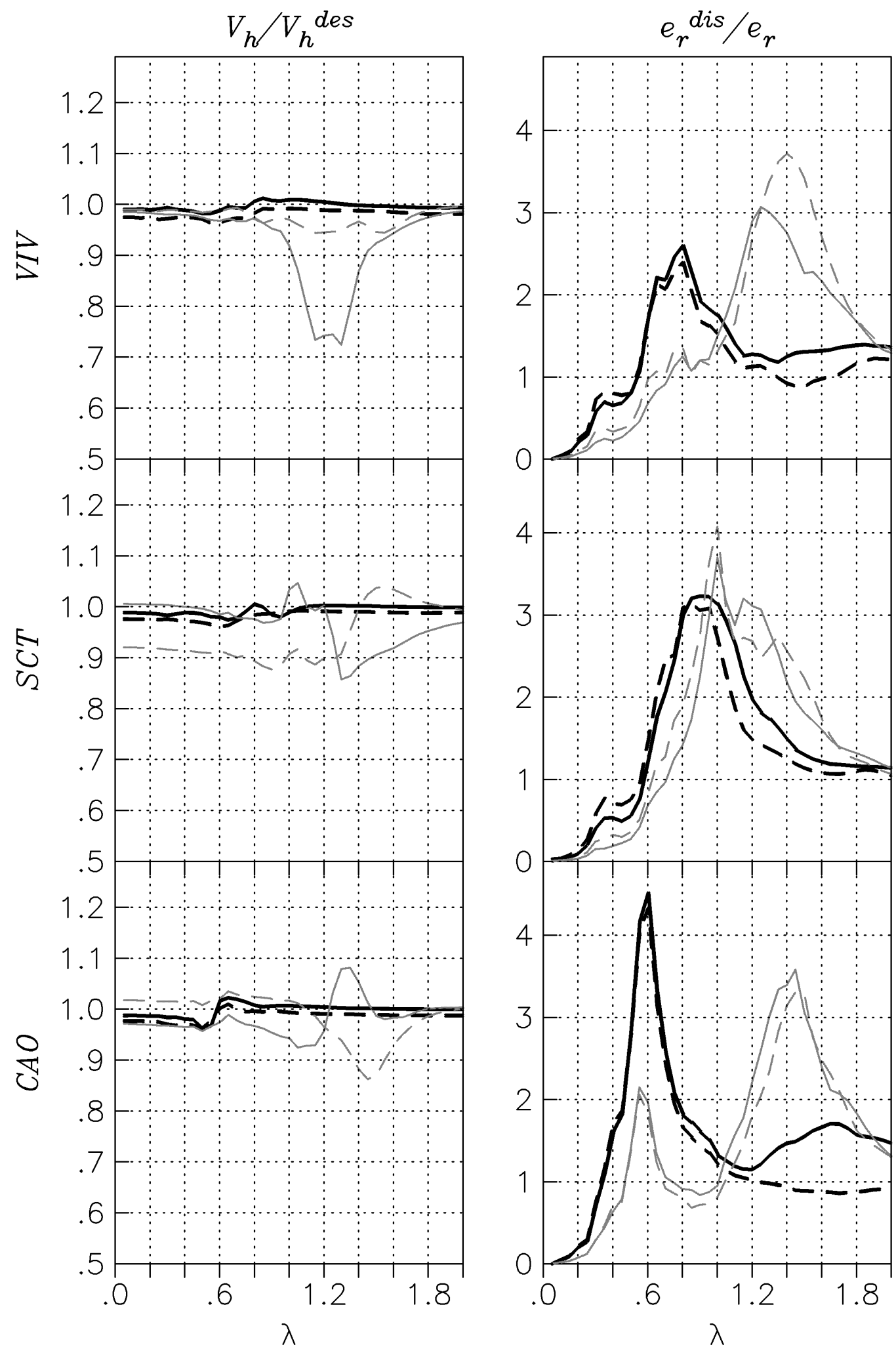

Figura 9. Resultados para osciladores con $D / R=0$ (líneas continuas) y $D / R=1$ (líneas a trazos) desplantados en un estrato de $H_{S} / R=2$ (líneas gruesas) y $H_{S} / R=5$ (líneas delgadas). 
En la fig. 13 se comparan los resultados para modelos con $H_{s} / R=5, D / R=0, T_{h}=2 s$ y $H_{e} / R=1$, variando las excentricidades estáticas, a saber: $e_{r} / R=0.05,0.10$ y 0.20 . Se observa que el valor de la excentricidad influye sólo en la amplitud de la respuesta. Se aprecia que si la excentricidad es muy pequeña, los valores de amplificación dinámica de la excentricidad que se pueden presentar son muy grandes, superiores a 4.5, y los valores del cociente de los cortantes son chicos, más de 3 veces lo señalado en las normas para el primer caso y sólo un 10\% para el segundo caso cuando se utiliza el registro de $S C T$, lo cual implica que muy probablemente un sistema estructural con las características descritas pueda ser subvaluado, todo dependerá de qué tanto compense la torsión accidental a la natural. Esta deficiencia en el reglamento no es grave, puesto que se presenta para excentricidades pequeñas lo que implica que se tengan momentos torsionantes también pequeños. Utilizando el mismo registro $(S C T)$ se presentan amplificaciones de la excentricidad de 2 y cociente de cortantes $50 \%$ cuando $e_{r} / R=0.2$, lo que probablemente tienda en este caso a sobrevaluar a sistemas estructurales estructuras con excentricidades grandes.

De los resultados obtenidos se observa que los parámetros que más influyen en la amplificación de dinámica tanto de la excentricidad como del cociente de cortantes, están la excentricidad y esbeltez estructural, y el espesor del estrato. La posición de los picos básicamente la determinan los periodos de traslación de la estructura y dominante del suelo. En las figs. 14 y 15 se presentan varios de los resultados obtenidos. En la fig. 14 se consideran estructuras con $T_{h}=1 s, H_{e} / R=1$ y 3, y $e_{r} / R=0.05,0.10$ y 0.20 desplantadas en estratos con $H_{s} / R=2,3$ y 5, y $T_{s}=3.5$, 2 y $0.6 s$ (registros de $C A O, S C T$ y $V I V$, respectivamente) y en la fig. 15 se presentan los resultados para estructuras con $T_{h}=0.5,2$ y $3 s, H_{e} / R=1$ y 3, y $e_{r} / R=0.05,0.10$ y 0.20 desplantadas en estratos con $H_{s} / R=2,3$ y 5, y $T_{s}=2 s$. En las gráficas se puede apreciar que la tendencia a agruparse de las curvas depende de los periodos del suelo y la estructura. Aunque la amplitud es determinante en el diseño estructural, y en cierta medida la consideran las actuales normas al involucrar a la excentricidad estructural en las fórmulas reglamentarias, para un diseño más racional se tendrá que considerar la relación de periodos de la estructura y el sitio donde se pretende construir, dado que la posición de los picos la determina este parámetro.

\section{CONCLUSIONES}

En este trabajo se analizó la respuesta de estructuras asimétricas de un nivel, modeladas por medio de un oscilador simple, sometidas al sismo de Michoacán del 19 de septiembre de 1985 registrado en las estaciones $V I V, S C T$ y $C A O$. El propósito fue investigar y destacar las condiciones para las cuales las recomendaciones de diseño por torsión propuestas en el reglamento son deficientes. Algunos autores ya han señalado cuáles estructuras no están adecuadamente protegidas por los reglamentos de algunas ciudades, entre ellos el de la ciudad de México. Aquí se confirman algunas de sus observaciones y se destacan otras que no habían sido contempladas, entre ellas el que no siempre se presentan las mayores amplificaciones dinámicas de la excentricidad cuando los valores de $\lambda$ son cercanos a uno. Estas se pueden presentar en valores tan grandes como $\lambda=1.4$, además en ocasiones se presentan varios picos (amplificaciones para distintos valores de $\lambda$ en una misma gráfica). 

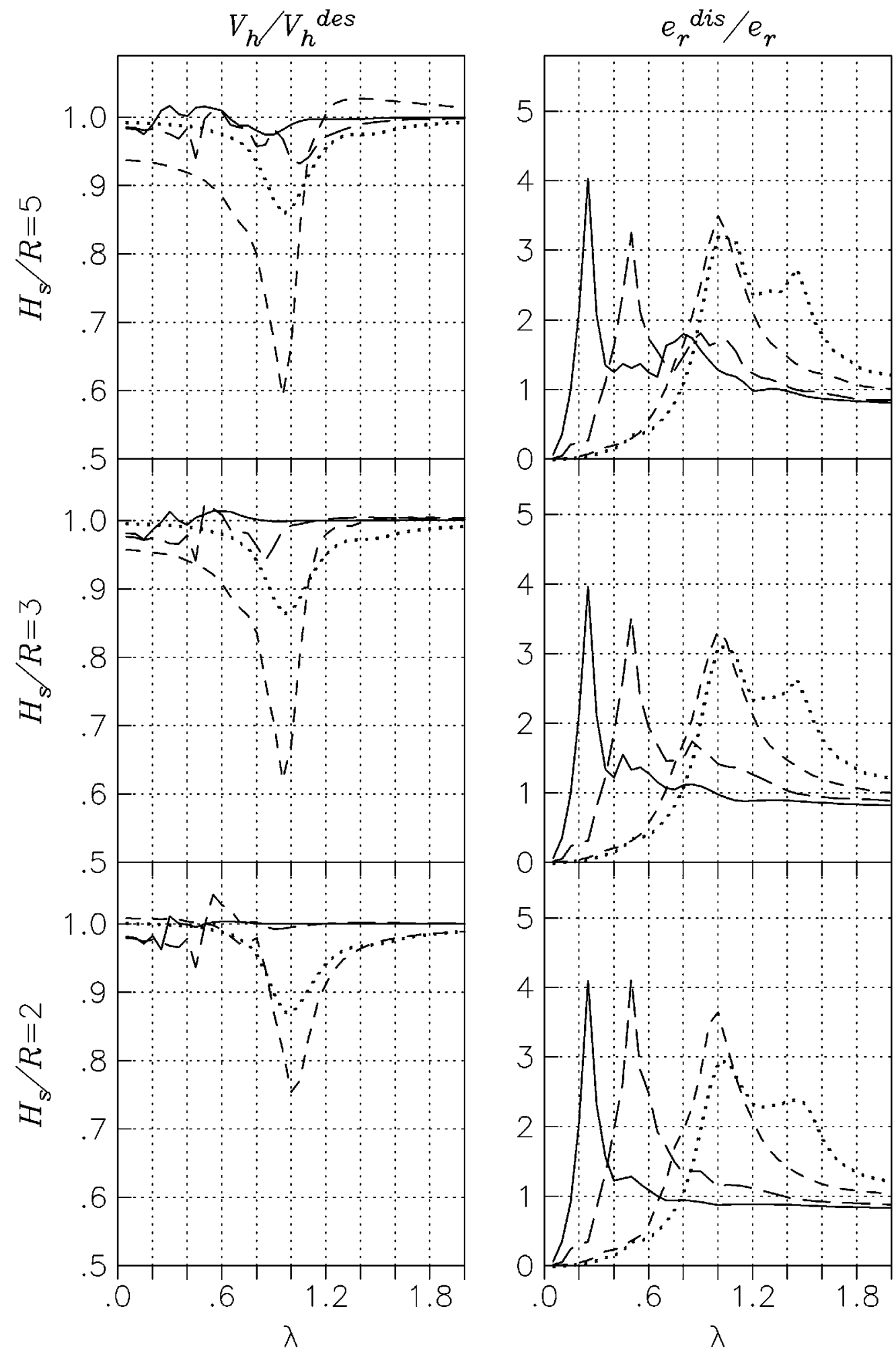

Figura 10. Resultados para osciladores con $T_{h}=0.5$ (línea continua), $T_{h}=1$ (trazos largos), $T_{h}=2$ (trazos pequeños) y $T_{h}=3 \mathrm{~s}$ (línea punteada) utilizando como señal de entrada el registro de $S C T$. 

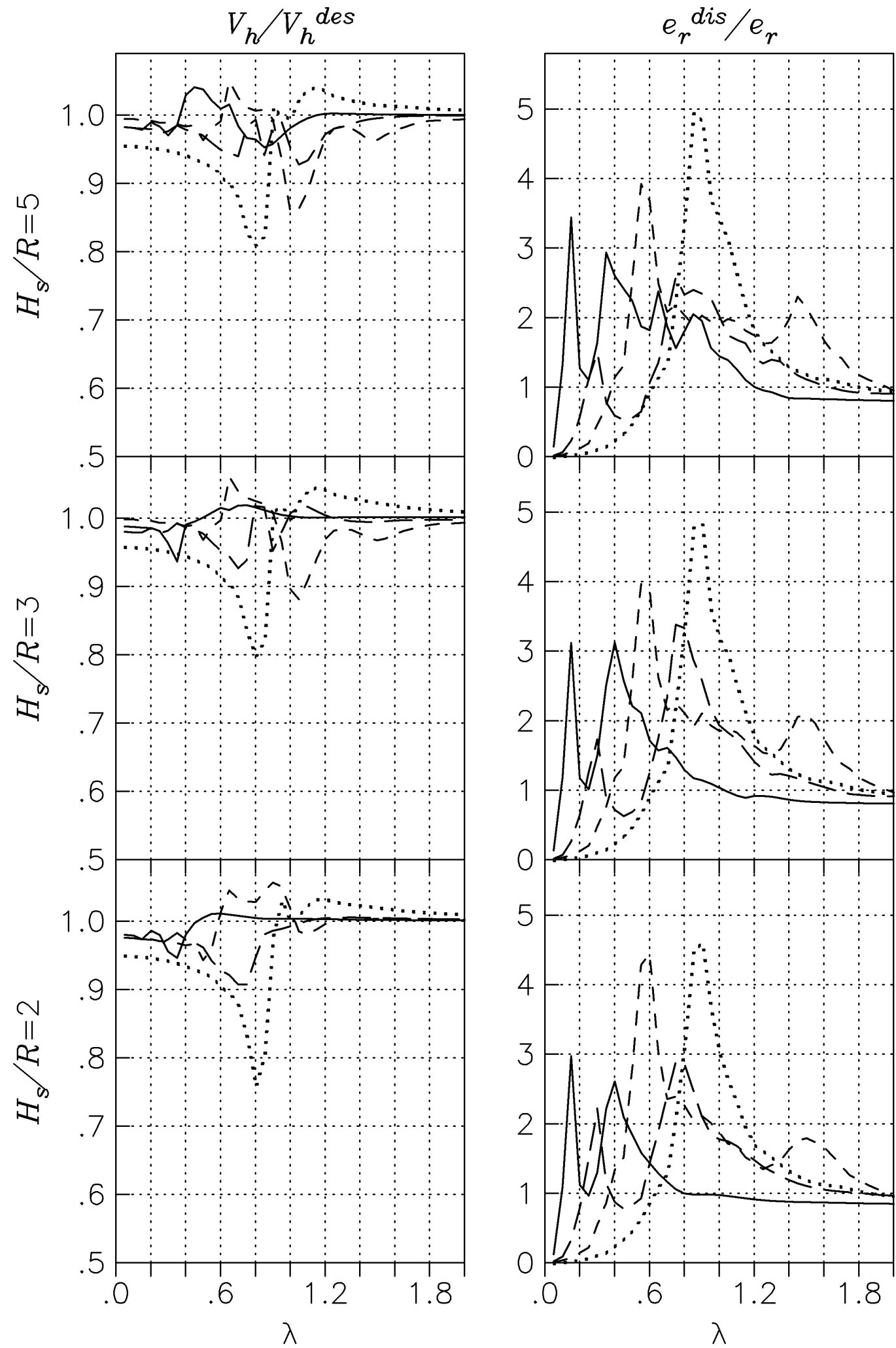

Figura 11. Resultados para osciladores con $T_{h}=0.5$ (línea continua), $T_{h}=1$ (trazos largos), $T_{h}=2$ (trazos pequeños) y $T_{h}=3 \mathrm{~s}$ (línea punteada) utilizando como señal de entrada el registro de $C A O$. 

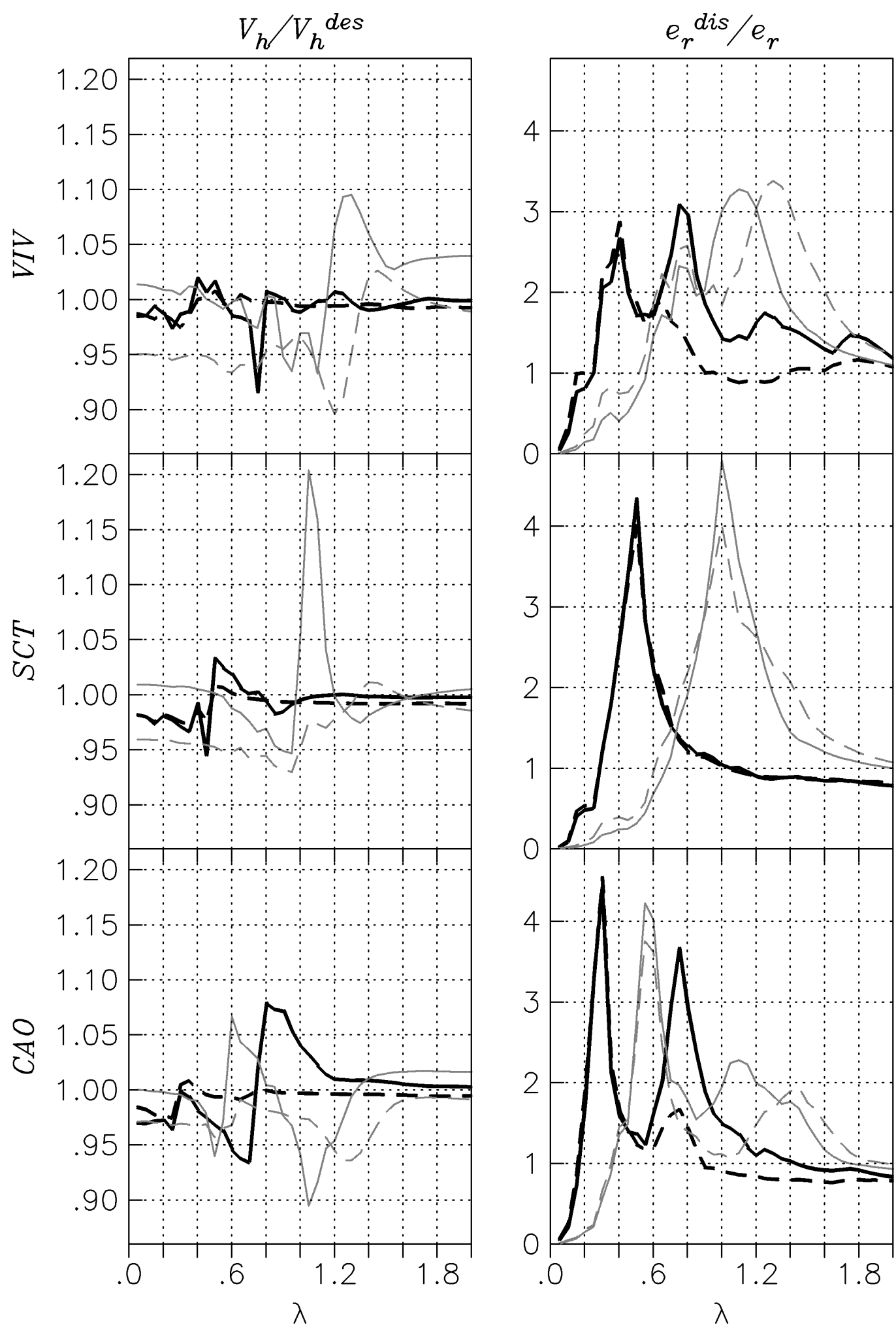

Figura 12. Resultados para osciladores con $H_{e} / R=1$ (líneas continuas) y $H_{e} / R=3$ (líneas a trazos), y $T_{h}=1$ (líneas gruesas) y $T_{h}=2 s$ (líneas delgadas). 

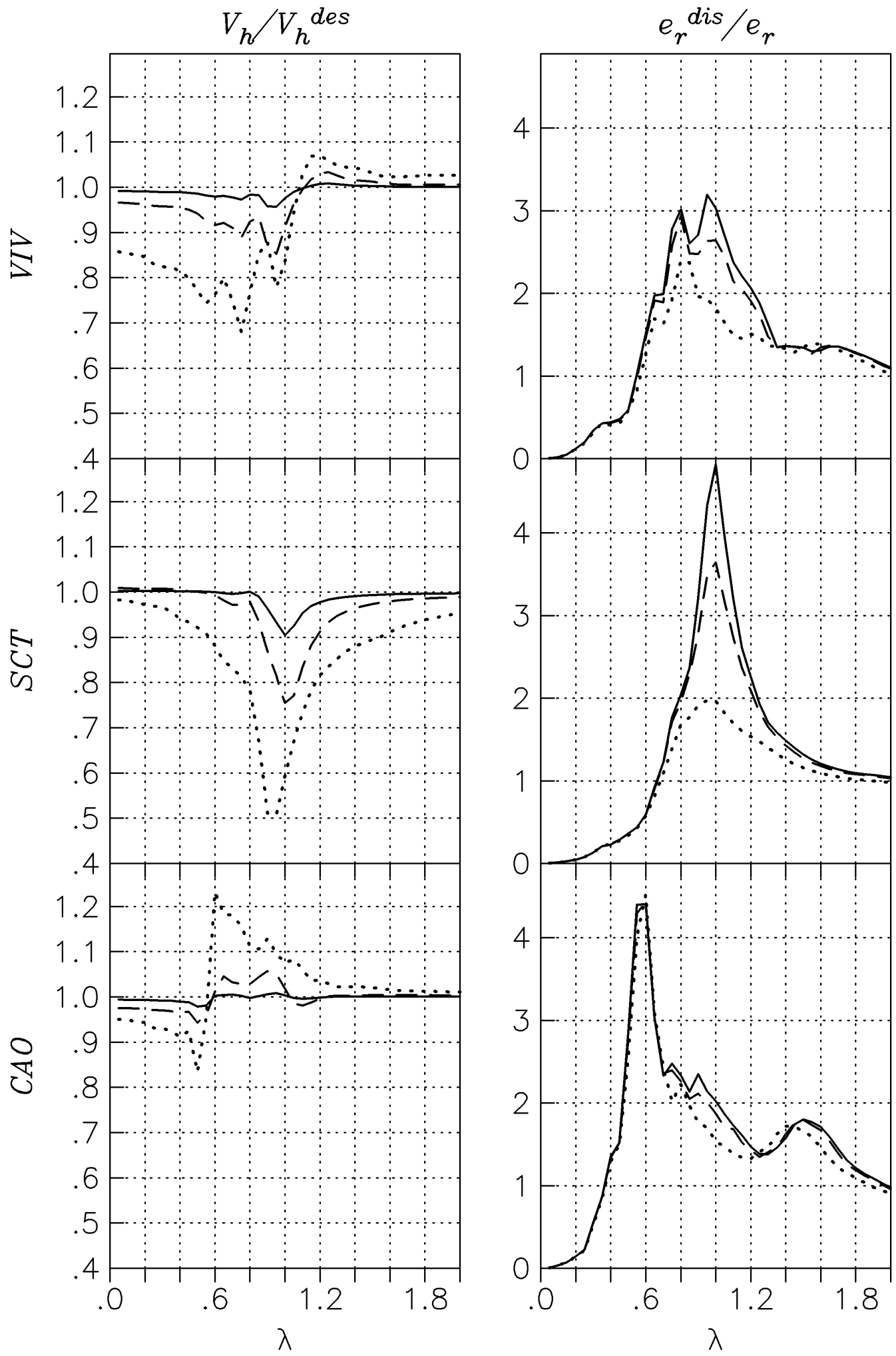

Figura 13. Resultados para osciladores con excentricidades $e_{r} / R=0.05$ (línea continua), $e_{r} / R=0.10$ (línea a trazos) y $e_{r} / R=0.20$ (línea punteada). 

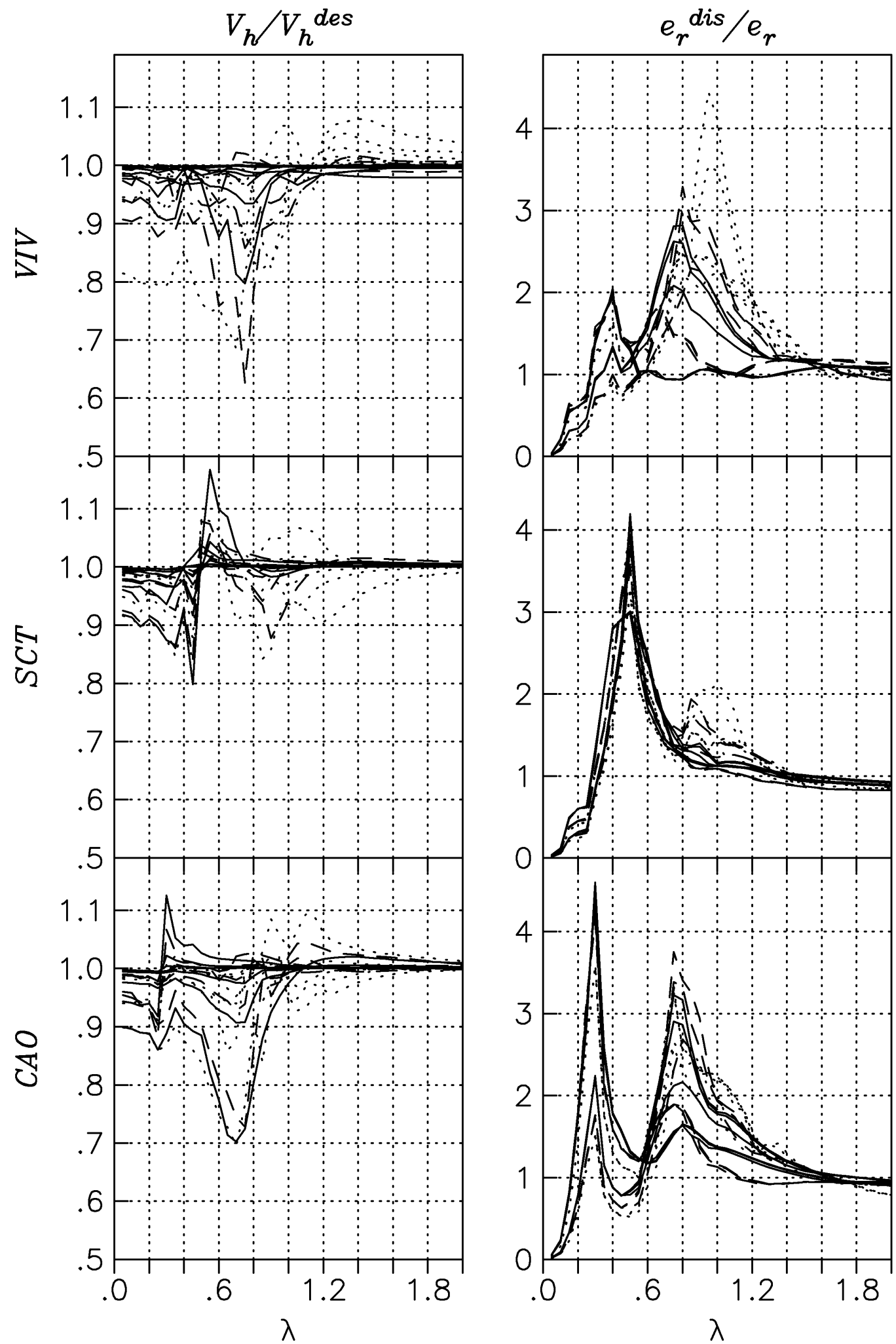

Figura 14. Resultados para osciladores con $T_{h}=1 s, H_{e} / R=1$ y 3, y $e_{r} / R=0.05,0.10$ y 0.20 en estratos con $H_{s} / R=2$ (línea continua), 3 (línea a trazos) y 5 (línea punteada), y $T_{s}=3.5,2$ y $0.6 s$. 

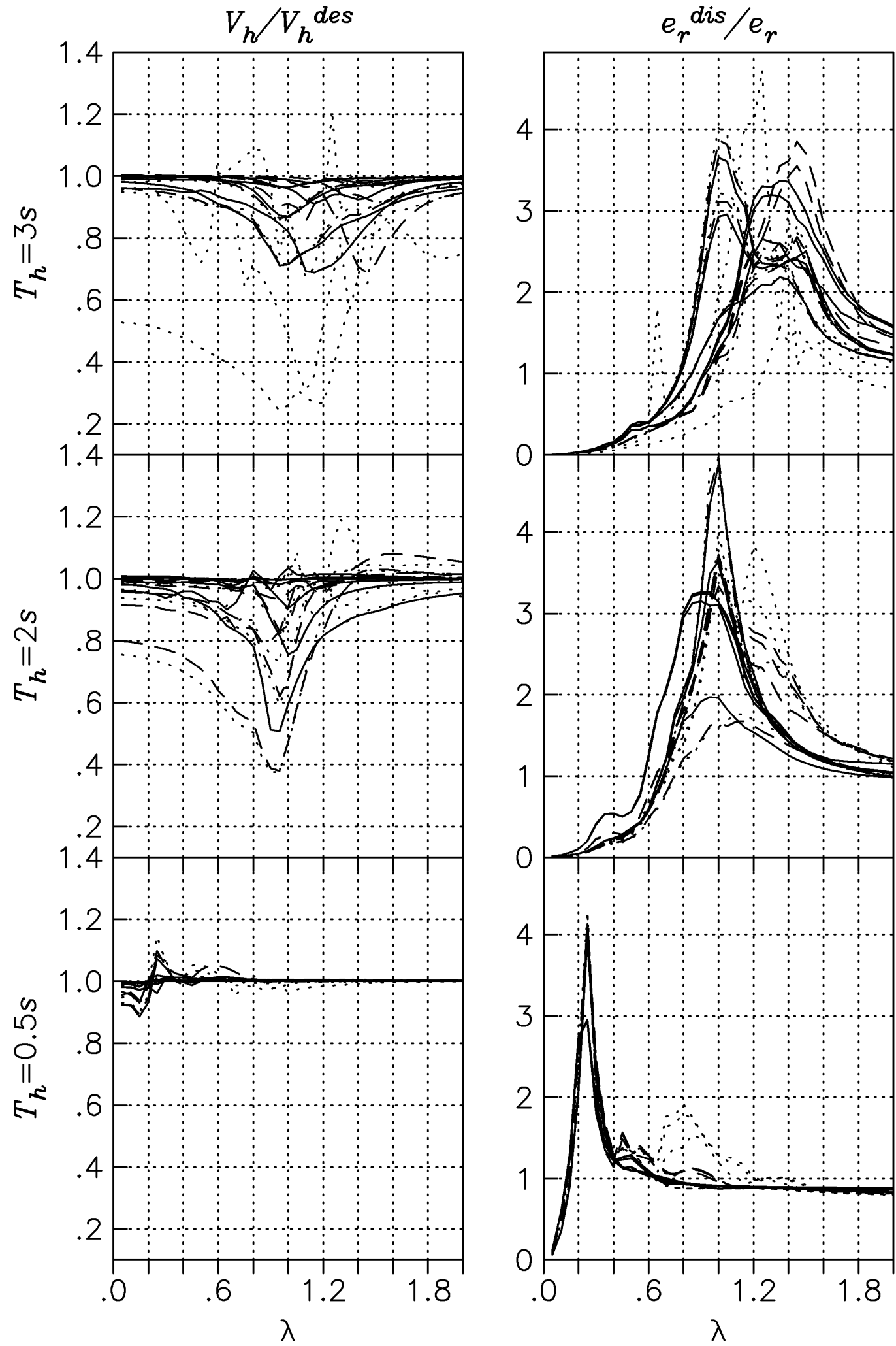

Figura 15. Resultados para osciladores con $H_{e} / R=1$ y 3 , y $e_{r} / R=0.05,0.10$ y 0.20 en estratos con $H_{s} / R=2$ (línea continua), 3 (línea a trazos) y 5 (línea punteada), y $T_{s}=2 \mathrm{~s}$. 
Se ha encontrado que las cimentaciones enterradas presentan casi el mismo comportamiento que las superficiales en cuanto a las amplificaciones dinámicas del cortante y la excentricidad. Esto implica que es válido considerar solamente cimentaciones superficiales cuando se llevan a cabo análisis de interacción con torsión. Esto solamente es aplicable a estratos que tengan un fuerte contraste de impedancias con el semiespacio. Para otros casos en donde la influencia del ángulo de incidencia de la excitación sea importante, el despreciar la interacción cinemática podría llevar a resultados que subestimen la respuesta estructural.

Como era de esperarse, la amplificación dinámica del cortante y la excentricidad tiene mayor relevancia para suelos blandos, aunque también se tienen valores de amplificación importantes en suelos moderadamente flexibles. Los parámetros que tienen mayor influencia en los resultados son la esbeltez, excentricidad estática y rigidez de la estructura, así como la flexibilidad del suelo. En general, se encontró que para estructuras con $\lambda \geq 1.5$ los criterios utilizados en el reglamento son adecuados o sobrestiman las amplificaciones dinámicas de la excentricidad. Para $\lambda<1.5$, estas amplificaciones se pueden alejar significativamente del valor propuesto en las normas, dando lugar a que el momento torsionante estático equivalente sea subvaluado en incluso más del $300 \%$ para algunos casos. En cuanto al cociente entre el cortante basal acoplado y el desacoplado, se presentan atenuaciones hasta más de un 45\% para excentricidades estructurales grandes. En su libro, Rosenblueth y Newmark (1971) previenen esto cuando al proponer las fórmulas empíricas que posteriormente se utilizaron en el reglamento, mencionan que '...dentro de las limitaciones impuestas con respecto a $K / k$, la excentricidad de diseño por piso es la más desfavorable de las cantidades

$e^{d i s}=\left\{\begin{array}{r}1.5 e+\alpha b \\ e-\alpha b\end{array}\right.$

donde $b$ es la dimensión de la planta del edificio y $\alpha$ es un coeficiente del orden de 0.05 a 0.1 '. En esta cita, $K / k$ se refiere a la relación de rigideces a la torsión sobre la de traslación y para poder aplicar este criterio $K / k>2.6 J_{\theta} / M-2 e^{2}$. Considerando estas limitaciones en el modelo aquí estudiado, las fórmulas en el reglamento serán válidas para valores de $\lambda$ mayores de 1.7. Para poder presentar criterios de diseño más adecuados, las modificaciones que se le realicen en un futuro a las actuales normas reglamentarias para el diseño de estructuras a la torsión, deberán tomar en cuenta parámetros relacionados con los periodos naturales de vibrar del suelo y de la estructura.

\section{RECONOCIMIENTOS}

Este estudio se realizó con apoyo parcial de CONACYT (proyecto número 27497U) y de DGAPA-UNAM (proyecto número IN104998). 


\section{REFERENCIAS}

Achenbach, J D (1976), Wave Propagation in Elastic Solids, North-Holland Publishing Company, Oxford.

Apsel, R y J E Luco (1976), “Torsional response of rigid embedded foundation”, ASCE Journal of Engineering Mechanics, pp. 957-971.

Bielak, J, H Sudarbo y D V Morse (1986), “Coupled lateral-rocking-torsional response of structures with embedded foundations due to SH-waves", Memorias, 3rd U.S. National Conference on Earthquake Engineering, Vol. II, August 24-28, Charleston, S. C., pp. 811-822.

Bozorgnia, Y y W K Tso (1986), "Inelastic earthquake response of asymmetric structures", ASCE Journal of Structural Engineering, Vol. 117, pp. 3762-3782.

Chandler, A M y G L Hutchinson (1987a), "Evaluation of code torsional provisions by a time history approach", Earthquake Engineering \& Structural Dynamics, Vol. 112, No. 2, pp. 383-400.

Chandler, A M y G L Hutchinson (1987b), "Code design provisions for torsionally coupled building on elastic foundation”, Earthquake Engineering \& Structural Dynamics, Vol. 15, pp. 517-536.

Chopra, A K y R K Goel (1991), "Evaluation of torsional provisions in seismic codes", ASCE Journal of Structural Engineering, Vol. 117, pp. 3762-3782.

Gaceta Oficial Del Distrito Federal (1995), "Secretaría de obras y servicios, Normas técnicas complementarias para diseño por sismo", Ciudad de México DF, 300, Tomo III.

Hahn, G D y X Liu (1994), "Torsional response of unsymmetric buildings to incoherent ground motions", ASCE Journal of Structural Engineering, Vol. 120, pp. 158-181.

Iguchi, M (1984), "Earthquake response of embedded cylindrical foundations to SH and SV waves", Memorias, 8th World Conference on Earthquake Engineering, San Francisco, California, pp. 1081-1088.

Luco, J E (1976a), "Torsional response of structures to obliquely incident seismic SH waves", Earthquake Engineering \& Structural Dynamics, Vol. 4, pp. 207-219.

Luco, J E (1976b), "Torsional response of structures for $S H$ waves: the case of hemispherical foundations", Bulletin of the Seismological Society of America, No. 66, pp. 109-123.

Newmark, N M (1969), "Torsion in symmetrical buildings", Memorias, 4th World Conference on Earthquake Engineering, Vol. I, A3, pp. 19-32.

Rosenblueth, E y J Elorduy (1969), "Responses of linear systems to certain transient disturbances", Memorias, 4th World Conference on Earthquake Engineering, Vol. I, A1, pp. 185196.

Newmark, N M y E. Rosenblueth (1971), Fundamentos de ingeniería sísmica, primera edición, Editorial Diana.

Tassoulas, J L y E Kausel (1983), "Elements for the numerical analysis of wave motion in a layered strata", International Journal of Numerical Methods in Engineering, No. 19, pp. 1005-1032. 
Tso, W K y T J Zhu (1992), "Design of torsionally unbalanced structural systems based on code provisions I: ductility demand", Earthquake Engineering \& Structural Dynamics, Vol. 21, pp. 609627.

Zhu, T J y W K Tso (1992), "Design of torsionally unbalanced structural systems based on code provisions II: strength distribution”, Earthquake Engineering \& Structural Dynamics, Vol. 21, pp. 629-644. 